\title{
Reseña de Lorenzano, Pablo y Oscar Nudler (eds.), El camino desde Kuhn. La inconmensurabilidad hoy, Madrid: Biblioteca Nueva, 2012, 431 pp.*
}

Review of Lorenzano, Pablo and Oscar Nudler (eds.), El camino desde Kuhn. La inconmensurabilidad hoy, Madrid: Biblioteca Nueva, 2012, 431 pp.

A principios de la década de 1960, Kuhn enunció por primera vez, en La estructura de las revoluciones cientificas (Kuhn 1962) (en adelante ERC), la tesis de la inconmensurabilidad. Por aquel entonces, dicha tesis se hallaba íntimamente relacionada con el problema de la posibilidad y los límites del conocimiento científico, o al menos así lo pensaron algunos de sus críticos. En ERC, una de las maneras en las que el autor explica el fenómeno de la inconmensurabilidad es trazando una analogía con la teoría de la percepción desarrollada por los psicólogos de la Gestalt. Desde ese punto de vista, el cambio de un paradigma a otro es semejante al cambio en la estructuración de lo que se percibe. "Los cambios de paradigma hacen que los científicos vean de un modo distinto el mundo al que se aplica su investigación. En la medida en que su único acceso a dicho mundo es a través de lo que ven y hacen, podemos estar dispuestos a afirmar que tras una revolución los científicos responden a un mundo distinto" (Kuhn [1962] 2006, p. 212). Es decir, en el nuevo paradigma los conceptos varían sus relaciones cambiando su significado. El resultado deriva en cierta incomunicación entre las dos escuelas en competencia, luego de un cambio científico radical: "la comunicación a través de la frontera marcada por la revolución es inevitablemente parcial" (Kuhn [1962] 2006, p. 266).

La primera formulación de la tesis de la inconmensurabilidad tenía la característica de ser amplia e incluir diferentes características del paradigma científico; no es casual que Kuhn identifique, repetidas veces, el objeto de la ciencia como mundo o naturaleza. En el capítulo X de ERC, titulado "Las revoluciones como cambios del concepto de mundo", la tesis de la inconmensurabilidad aparece formulada de manera vaga y algo ambigua. Ciertamente, las siguientes cuatro afirmaciones extraídas del texto mencionado (Kuhn [1962] 2006) lo evidencian: a) "los cambios de paradigma hacen que los científicos vean de un modo distinto el mundo al que se aplica su investigación" (p. 212); b) "tras una revolución los científicos responden a un mundo distinto" (p. 212); c) "aunque el mundo no cambie con un cambio de paradigma, tras él el científico trabaja en un mundo distinto" (p. 227) y d) "sea lo que sea lo que ve el científico después de una revolución sigue mirando el mismo mundo" (p. 238). Los enunciados a) y b) aluden a la idea de que, en la medida en que los paradigmas sean los marcos -que inclu-

\footnotetext{
* Recibido: 2 de Febrero de 2013. Aceptado: 27 de Marzo de 2013.

Metatheoria 3(2) (2012): 109-117. ISSN 1853-2322.

(c) Editorial de la Universidad Nacional de Tres de Febrero. Publicado en la República Argentina.
} 
yen las herramientas, por ejemplo- dentro de los cuales puede ser interpretado un problema científico, entonces el científico que trabaja dentro de un paradigma crea, de alguna manera, su propio mundo de investigación. La derivación inmediata es el problema de la objetividad del mundo externo, independiente del modo de conocimiento que se tenga de él. Varios críticos han apuntado al relativismo que conllevarían las tesis mencionadas. Otros han enfocado esta idea bajo la interpretación de que la ciencia no trabaja con datos puros y que las investigaciones siempre contienen la carga de las teorías o, en el caso de Kuhn, de los paradigmas desde los cuales se investiga. Sin embargo, las proposiciones c) y d) son contrarias a la interpretación anterior. Kuhn parece admitir la existencia de un mundo independiente y que permanece estable a pesar de los cambios revolucionarios. Sin embargo, más allá de las ambigüedades que puedan notarse en ciertas sentencias aisladas, la sustancia del capítulo mencionado está en los ejemplos. Allí, el análisis de la idea de inconmensurabilidad, lejos de mostrarse contradictorio, es tratado de manera clara y precisa.

Amén de los acuerdos y desacuerdos con la tesis de la inconmensurabilidad en su primera formulación, lo cierto es que no pocos filósofos se ocuparon de la polémica. Y no solo los pertenecientes al ámbito de la filosofía de las ciencias naturales. A decir verdad, desde su primera formulación ha sido extendida a otras áreas como la filosofía de las ciencias sociales, la filosofía del lenguaje y de la mente, la semántica, etc. Un ejemplo de ello es el planteo de Donald Davidson (1974) en el que se ataca la posibilidad misma de formular el problema de la inconmensurabilidad, brindando razones para sostener que la mencionada tesis es insostenible.

Casi siete años después de su primera formulación y luego de las polémicas suscitadas, Kuhn escribe su Posdata, donde aclara algunas de sus tesis principales. Ahora, la inconmensurabilidad -que pasa a denominarse inconmensurabilidad local- se enfoca principalmente en el problema de la traducción entre términos de paradigmas en competencia. Esto es, la idea de que los problemas de traducción no afectan a todos los conceptos del paradigma sino solo a algunos. En dicho texto, Kuhn reconoce que la traducción de los términos de una teoría a otra nunca es completa, aunque no por ello imposible. De la tesis de Kuhn, que afirma que no existe diccionario de traducción neutral que permita pasar totalmente de un lenguaje a otro todos los términos implicados, no se desprende la imposibilidad de la comunicación entre los científicos que trabajan bajo paradigmas distintos. De hecho, el autor señala que "lo que pueden hacer los que se ven envueltos en una ruptura de la comunicación es reconocerse mutuamente como miembros de diferentes comunidades lingüísticas y hacerse entonces traductores" (Kuhn [1962] 2006, pp. 341-342).

Hasta sus últimos días Kuhn continuó desarrollando y precisando su concepto de inconmensurabilidad. Y así lo hicieron varios pensadores desde su primera formulación. En este sentido, es notable el trabajo de la concepción estructuralista de la ciencia (Balzer, Moulines \& Sneed 1987) que retoma varias de las tesis centrales de Kuhn, entre ellas la de la inconmensurabilidad, resignificando su 
sentido a la luz del programa estructuralista para la ciencia. De este modo, propone un modo de representar las teorías científicas, desechando la distinción entre términos teóricos y términos observacionales propia de la concepción heredada y proponiendo en su lugar la distinción entre términos T-teóricos y términos T-no-teóricos, es decir, relativos a determinada teoría científica (T). Bajo esta óptica, el programa estructuralista lleva adelante una reformulación de la tesis de la inconmensurabilidad entre términos correspondientes a diferentes teorías. En relación con la inconmensurabilidad teórica, sostienen que, en su versión más simple, "los términos que no pueden ser traducidos se identifican con los términos T-teóricos y aquellos que mantienen su significado serán identificados con los términos T-no-teóricos" (Lorenzano 2008, p. 247). Bajo esta óptica, la inconmensurabilidad permite dar cuenta de las rupturas y continuidades entre teorías a lo largo de su historia. Un desarrollo en este sentido es el esclarecimiento de la historia de la genética realizada por Pablo Lorenzano (2008), cuya versión ampliada y revisada es parte de la presente compilación.

Llegados a este punto huelga decir que el presente volumen contiene una serie de artículos de una variedad de filósofos de las ciencias de habla hispana nucleados alrededor de la cuestión de la inconmensurabilidad. El abordaje es realizado desde diferentes perspectivas o áreas de conocimiento, lo cual vuelve el texto aun más enriquecedor. Está conformado por doce artículos que resumiremos a continuación en sus tesis y objetivos principales.

El primero de ellos es "Inconmensurabilidad y dominios de aplicación" (pp. 27-65), donde Otávio Bueno analiza detalladamente los dominios de aplicación de las teorías científicas aceptando que aquello que es tomado como dominio de aplicación en determinado momento de la teoría no está exento de cambios. La estabilidad llega, paradójicamente, cuando una teoría rival sustituye enteramente a la anterior, aclarándose así su dominio de aplicación. Comprender los dominios de aplicación de las teorías es comprender a su vez las características del cambio científico; aquí es donde el autor introduce el análisis de la inconmensurabilidad en tanto asociada a los cambios en los dominios de aplicación. El concepto de inconmensurabilidad que maneja el autor se centrará en el problema de la inexistencia de "standards comunes de evaluación para determinar la referencia de ciertos términos teóricos" (p. 28). Así, determinar la referencia de tales términos dependerá de los dominios de aplicación considerados. Uno de los problemas analizados por el autor es la consecuencia para las interpretaciones realistas de aquellos términos que permanecen opacos en la determinación de su referencia. Esto es, cómo determinar los términos teóricos sin que dicha elección sea arbitraria. La solución ofrecida por Bueno provee una noción de dominio de aplicación en términos empiristas, es decir, que visibilice la práctica científica y reconozca la presencia de la inconmensurabilidad, pero que no derive en conclusiones relativistas. Los instrumentos científicos ocuparán un lugar central en la solución propuesta por el autor.

En "Inconmensurabilidad, comparabilidad empírica y escenas observacionales” (pp. 67-118), José A. Díez analiza desde una perspectiva lingüística y desde 
una modelo-teórica la posibilidad de elucidar la comparabilidad y rivalidad empírica. Su conclusión es que desde ninguno de los puntos de vista mencionados es posible tal determinación de manera satisfactoria si solo se recurre a los conceptos de las teorías que se usan en la formulación de sus leyes. Siguiendo a Díez, los problemas relativos a la inconmensurabilidad en tanto la posibilidad de comparar teorías en competencia no pueden ser resueltos por el solo hecho de sustituir los marcos lingüísticos por los modelo-teóricos. El autor asevera que "para resolver dichos problemas se hace necesaria la referencia a entidades distintas de las referidas por los conceptos centrales de la teoría en cuestión" (p. 105). El análisis de Díez comienza haciendo una serie de precisiones lingüístico-conceptuales: inconmensurabilidad débil y fuerte, inconmensurabilidad teórica y empírica y comparabilidad, rivalidad e inconmensurabilidad no trivial. El paso siguiente de su artículo se centra en la exposición y discusión de las tesis centrales de Kuhn y Feyerabend sobre la cuestión de la inconmensurabilidad, mostrando sus límites. Seguidamente, expone el análisis modelo-teórico estructuralista en su versión estándar y muestra las dificultades para resolver el problema de la inconmensurabilidad en los términos recortados por el autor. Finalmente, en su conclusión al problema de la elucidación correcta de la rivalidad empírica, propone la apelación a elementos conceptuales distintos de los usados por las teorías en la formulación de sus leyes. El autor brinda una serie de pistas para identificar dichos elementos.

En "Comparación epistémica de teorías inconmensurables, sin fundamentismo” (pp. 119-170), José L. Falguera cuestiona la carga teórica de la percepción $y$, por ende, que exista inconmensurabilidad perceptual. Falguera entiende que partes de teorías inconmensurables son igualmente comparables. Así, las teorías inconmensurables tratan sobre porciones del mundo compartidas. Ante la inconmensurabilidad perceptual, el autor sostiene que las experiencias perceptuales de sujetos diferentes, bajo las mismas condiciones, coinciden en contenido, con independencia de las creencias o teorías que asuman. Falguera lleva adelante un análisis de las condiciones ontosemánticas para la comparabilidad de teorías pretendidamente inconmensurables. Este análisis lo lleva a establecer que dos teorías inconmensurables que tratan sobre características del mundo compartidas se dan por medio del lenguaje no común a ambas y por medio de experiencias preceptuales coincidentes.

En "Consideraciones críticas sobre la tesis de la inconmensurabilidad de Kuhn” (pp. 171-193), Luis Fernández Moreno se propone llevar adelante dos líneas críticas en relación con la tesis de la inconmensurabilidad de teorías planteada por Kuhn. Teniendo en cuenta las diferentes justificaciones del concepto expuestas por el mismo Kuhn, el autor llevará adelante el análisis a partir de una línea centrada en la idea de traducción y de otra centrada en la noción de referencia. El artículo consta de dos partes. En la primera Fernández Moreno aborda el análisis de la polémica entre Kuhn y la teoría causal de la referencia, concluyendo que el primero no otorga una justificación suficiente de la tesis del cambio de referencia. Por su parte, otra tesis emparentada con la recién menciona- 
da, aunque más débil, se presenta como más plausible. Esto es, la tesis de que las teorías pueden conllevar cambios de referencia. En la segunda parte del artículo, y luego del análisis pormenorizado de la noción de traducción de Kuhn, el autor concluye que la cuestión de la traducción, expuesta por Kuhn en su tesis de la inconmensurabilidad conduce a un dilema: la tesis resulta o trivial o falsa.

En "Patrones de descubrimiento, inconmensurabilidad y clases. Un caso ejemplar" (pp. 195-224), Adriana Gonzalo analiza la temática de la inconmensurabilidad con relación a las taxonomías científicas. Así, primeramente aborda la noción de inconmensurabilidad desarrollada por Kuhn en distintas obras. Luego, se realiza un análisis de un caso histórico: la organización taxonómica del mundo animal en el siglo XVIII en la región del Litoral Argentino. Para la autora, dicho caso pone en evidencia cómo los nuevos descubrimientos científicos son vistos y conceptualizados a partir de un sistema cultural heredado y de la concepción del mundo hegemónica en una comunidad intelectual. Apoyada en los argumentos brindados a partir del análisis del ejemplo, la autora otorga elementos de juicio en favor de la tesis de la inconmensurabilidad teórica de Kuhn en su versión débil, analizada en la primera parte del trabajo.

En " ¿Es compatible la idea de inconmensurabilidad no trivial con la de progreso científico? Algunas razones a favor de la compatibilidad” (pp. 225-264), Juan Manuel Jaramillo Uribe analiza en primer lugar el problema del progreso científico en los casos de teorías separadas por una revolución científica. Muestra cómo el progreso científico en los casos en los que se produce una sustitución teórica ha sido objeto de controversia tanto para la concepción heredada de las ciencias como para la llamada concepción estructuralista. Sin embargo, para el autor, esta última otorga de manera exitosa un marco riguroso para el tratamiento del mencionado problema apelando a conceptos tales como "reducción exacta (Adams-Sneed), reducción aproximativa (Stegmüller), explicación aproximativa (Scheibe) o aproximación interteórica (Moulines) y al haberse ocupado de instanciar dichas nociones valiéndose de teorías concretas" (pp. 258-259). Frente al dilema -tal como lo plantea Putnam- de aceptar la existencia de dos proposiciones irreconciliables -la que asevera un progreso acumulativo del conocimiento objetivo y la que afirma la existencia de cambios radicales en la evolución del conocimiento científico y, por ende, la imposibilidad de un progreso científico-, Uribe propone una tercera salida: la de quienes sostienen que, a pesar de los cambios radicales en la evolución de las teorías, es posible continuar hablando de progreso científico. Para ello es necesario abandonar una concepción enunciativa de las teorías científicas y adoptar la visión estructuralista. Con esta última concepción, el progreso científico no desaparece, aunque las teorías que se sucedan en el tiempo categoricen el mundo de manera distinta y hagan uso de diferentes leyes fundamentales y espaciales, pues el criterio de ordenación de teorías no depende de una pretendida uniformidad enunciativa o legaliforme. Finalmente, el autor concluye qu,e desde un análisis estructuralista de teorías, la idea de progreso científico no se presenta como incompatible respecto de la de inconmensurabilidad. 
En “Acerca de la inconmensurabilidad” (pp. 265-288), César Lorenzano lleva adelante una minuciosa caracterización de la tesis de la inconmensurabilidad. Parte de los orígenes matemáticos del concepto hasta llegar a su caracterización kuhniana. Luego, examina argumentos a favor y en contra de ella. Los argumentos en contra coinciden en que la noción de inconmensurabilidad imposibilita la traducción entre teorías y, por ende, su comparación. El autor muestra cómo dichos argumentos tienen una relación de similitud con aquellos que sostienen la imposibilidad de la reducción de los términos teóricos a los observacionales y la imposibilidad de la traducción perfecta. Seguidamente, y a partir del análisis de dos teorías contrapuestas pertenecientes al campo de la medicina, se propone mostrar la tesis de la inconmensurabilidad como una tesis razonable tanto desde el punto de vista del sentido común como desde el punto de vista científico. Así, el autor afirma que las teorías pueden ser comparadas y seleccionadas, aunque los términos y las estructuras teóricas de dos teorías sean inconmensurables. Finalmente, el autor muestra cómo una concepción de inconmensurabilidad que suponga la imposibilidad de traducción y comparación está asentada en un esencialismo platonista no asumido por sus defensores y que solo una visión nominalista desde el punto de vista epistémico es consistente con la práctica científica efectiva y el uso de las teorías científicas.

Pablo Lorenzano, en su texto "Estructuras y aplicaciones intencionales: Inconmensurabilidad teórica y comparabilidad empírica en la historia de la genética clásica" (pp. 289-350), pretende capturar y precisar la idea de "que entre las propuestas de Mendel, de los 'redescubridores' -De Vries, Correns y Tschermak-, de Baterson y colaboradores y de Morgan y discípulos se dan ciertas discontinuidades y rupturas, como la de las continuidades que permitirían hablar de una historia oficial de la genética” (p. 294). El autor lleva a cabo el análisis desde un punto de vista diacrónico y cinemático. Para ello, en una primera parte da cuenta de aquellas condiciones que se consideran necesarias para hablar de identidad de teorías. En segundo lugar, discute la noción de inconmesurabilidad de Kuhn. Y, en un tercer momento, relaciona el mencionado concepto con la concepción estructuralista de las teorías. Aquí Lorenzano introduce los conceptos de inconmensurabilidad teórica y comparabilidad empírica equivalentes a las nociones de inconmensurabilidad parcial y base semántica común propuestas por Kuhn. A continuación, presenta a grandes rasgos una exposición de la historia de la genética analizando cada uno de los desarrollos de los autores anteriormente nombrados. Finalmente, las categorías propias del estructuralismo metacientífico introducidas con anterioridad -inconmensurabilidad teórica y comparabilidad empírica- son aplicadas a los desarrollos de la historia de la genética, mostrando así tanto las continuidades como las discontinuidades en los diferentes niveles conceptual y aplicativo.

En "Inconmensurabilidad y metáfora" (pp. 351-365), Oscar Nudler nos propone un recorrido en el análisis del concepto de inconmensurabilidad distinto al planteado por la literatura habitual sobre la noción introducida por Kuhn y Feyerabend. La propuesta del autor se centra en la inconmensurabilidad existen- 
te entre teorías que están estructuradas en base a metáforas diferentes. Un ejemplo es el de las teorías mecánica y orgánica estructuradas por la metáfora de la máquina y la del organismo, respectivamente. A diferencia de la inconmensurabilidad kunhiana, asentada sobre la diferencia de significado entre términos, la inconmensurabilidad que trabaja Nudler se centra en "la diferencia entre las metáforas presupuestas en las respectivas teorías" (p. 352) En la primera parte del trabajo, el autor analiza la noción de metáfora en su aspecto cognoscitivo, mostrando cómo luego de su rechazo por varios siglos se logró un amplio consenso sobre el tema. En la segunda parte, introduce el concepto de proyección metafórica, concepto que permite extender la aplicación del significado de ciertos términos más allá de los límites pretendidamente originales. La caracterización del concepto mencionado se completa con una serie de ejemplos tomados del lenguaje ordinario. Así, analiza términos que dan cuenta de características espaciales y muestra cómo funcionan de estructuradores para amplias porciones del lenguaje natural. De los ejemplos del lenguaje natural, el autor pasa a analizar cómo dichas metáforas estructuradoras se expanden también al ámbito de los lenguajes teóricos. Las teorías contemporáneas de la mente constituyen los primeros casos elegidos por el autor para ejemplificar el carácter estructurante de las metáforas, luego el análisis se extiende también a otros dominios. Finalmente, Nudler aplica el desarrollo de las secciones anteriores al problema de la inconmensurabilidad y concluye tanto la posibilidad de la comunicación entre teorías metafóricamente inconmensurables como la posibilidad del aprendizaje mutuo entre las teorías mediante el diálogo de metáforas.

En "Recuperando la inconmensurabilidad axiológica" (pp. 367-382), Ana Rosa Pérez Ransanz aborda la cuestión de la legitimidad del concepto de inconmensurabilidad, esto es, pretende "determinar si se trata de un problema efectivo de la actividad científica, si se trata de un problema efectivo pero sobreestimado por la comunidad científica o si constituye un seudo problema” (p. 367). La autora se inclina por la primera de las opciones. La inconmensurabilidad representa un problema real de la investigación científica; sin embargo, destaca que el tipo de inconmensurabilidad estimado por la mayoría de los estudiosos reduce el concepto a una cuestión semántica: el cambio conceptual en las teorías evidenciado por los fracasos en la traducción y problemas de comunicación entre teorías en competencia. De esta manera, Pérez Ransanz pretende rescatar otro sentido de inconmensurabilidad, tan importante como la otra para comprender la actividad científica, denominada inconmensurabilidad axiológica y que, según la autora, está igual de presente que la inconmensurabilidad semántica, al menos en lo que respecta a ERC. Así, la autora lleva adelante un análisis de esta segunda inconmensurabilidad con el fin de mostrar sus implicaciones en el proceso de comparación y elección de teorías. "La intención es mostrar que dicha inconmensurabilidad permite identificar mecanismos de formación de consensos en la comunidad científica y ofrecer razones que aporten al carácter racional de la elección de teorías" (p. 368). Asimismo, muestra que ambas dimensiones de la inconmensurabilidad fundamentan un pluralismo epistemológico, lejos de posiciones relativistas. 
En "La tesis de la inconmensurabilidad y el desarrollo de la física" (pp. 383 398), Andrés Rivadulla sostiene que, aunque la inconmensurabilidad en un principio se identificaba con una serie de problema diversos, a partir de los años ochenta Kuhn determina su significado en términos semánticos: la inconmensurabilidad en tanto la imposibilidad de la traducción entre conceptos. "La inconmensurabilidad sería de esta manera el cambio de significado de términos homónimos usados en distintas teorías científicas" (pp. 383-384). Los términos de teorías inconmensurables resultan de esta manera incomparables entre sí. La tesis de la dependencia contextual del significado, proveniente de la filosofía del lenguaje, se encuentra a la base del mencionado concepto de inconmensurabilidad. Esto es así pues implica que, para determinar el significado de un término, es necesario recurrir a su contexto teórico determinado y, si los contextos son distintos, entonces lo es también el significado de los términos. De esta manera, incluso tratándose de términos homónimos, estaríamos frente a la imposibilidad de traducirlos entre sí y las teorías que los contienen resultarían incomparables. Para el autor, las ideas de Kuhn y Feyerabend se separan de la física y las consecuencias de la inconmensurabilidad son menos adversas para la racionalidad de la ciencia de lo que parecen, a juzgar por sus repercusiones. El análisis de la pretendida inconmensurabilidad de los términos teóricos flogisto, planeta y masa será llevado a cabo por Rivadulla con el fin de evaluar la viabilidad de la tesis filosófica de la inconmensurabilidad. Finalmente, el autor muestra cómo "el análisis dimensional hace posible el uso simultáneo de teorías inconmensurables dentro de un mismo dominio" (p. 384), lo cual constituye un argumento en contra de la tesis filosófica de la inconmensurabilidad.

En "Hacking Kuhn: los límites de la inconmensurabilidad" (pp. 399-431), Mauricio Suárez lleva adelante una defensa de las tesis de Kuhn desde la perspectiva de lo que se denomina nuevo experimentalismo o escuela de Stanford. El autor sostiene que una lectura del tipo que lleva adelante dicha escuela permite disolver algunas de las principales objeciones que se han presentado en contra de las tesis kuhnianas. Las objeciones sobre las que se concentra el artículo son cuatro: pesimismo epistemológico, irracionalismo metodológico, relativismo semántico e idealismo. Si bien el texto no se concentra en la tesis de la inconmensurabilidad, Suárez sostiene "que la conjunción lógica de las objeciones antes mencionadas se presenta como equivalente de la tesis de la inconmensurabilidad, desde un marco que el autor denomina la dicotomía clásica" (p. 400). Dicho marco valida el conocimiento científico en tanto conocimiento teórico, susceptible de confrontación o verificación a partir de los datos de la experiencia. De esta manera, el problema de la inconmensurabilidad será el problema de la inconmensurabilidad de los paradigmas teóricos. Desde el punto de vista del nuevo experimentalismo, la tesis de la inconmensurabilidad es pensada como dos tesis lógicamente independientes la una de la otra: "la inconmensurabilidad de las teorías científicas y la inconmensurabilidad de las diversas formas de conocimiento experimental" (p. 401). A esta última tesis se opone abiertamente el nuevo experimentalismo al rechazar la dicotomía tradicional y proponer un modelo científi- 
co con una estructura de tres niveles: teorías y datos observacionales en los extremos y una compleja serie de modelos de los fenómenos que funcionan como mediadores entre las teorías y los datos. De esta manera, el autor afirma que, en el marco del nuevo experimentalismo, las objeciones antes mencionadas a la tesis de la inconmensurabilidad ya no se derivan, siendo el marco de conocimiento científico asumido lo que determina los límites de la tesis.

La presente obra aporta un exhaustivo análisis de uno de los problemas esenciales de la filosofía de las ciencias. La exposición clara, sistemática y rigurosa nos brinda un panorama general ordenado que nos permite abordar debates filosóficos del presente a partir del pasado y desde del presente hacia el pasado. Quien quiera adentrarse en las discusiones contemporáneas sobre los viejos y los nuevos problemas suscitados a partir de la tesis de la inconmensurabilidad no puede obviar el presente libro, que traza el camino desde Kuhn hasta nuestros días.

Sonia López Hanna

Universidad Nacional de Mar del Plata / Universidad Nacional de Tres de Febrero / Conicet

Bibliografía

Balzer, W., Moulines, C. U. y J. D. Sneed (1987), An Architectonic for Science. The Structuralist Program, Dordrecht: Reidel. (Versión en español: Una arquitectónica para la ciencia. El programa estructuralista, Lorenzano, P. (trad.), Bernal: Universidad Nacional de Quilmes, 2012.)

Davidson, D. (1990), "Sobre la idea misma de un esquema conceptual”, en Davidson, D., Ensayos sobre la verdad y la interpretación, Barcelona: Gedisa, pp. 189-203.

Kuhn, T. S. (1962), The Structure of Scientific Revolutions, Chicago: University of Chicago Press. (Versión en español: La estructura de las revoluciones científicas, Solís, C. (trad.), México: Fondo de Cultura Económica, 2006.)

Lorenzano, P. (2008), "Inconmensurabilidad teórica y comparabilidad empírica: el caso de la genética clásica”, Análisis Filosófico XXVIII(2): 239-279. 